\title{
Telomere length, COPD and emphysema as risk factors for lung cancer
}

To the Editor:

Telomeres are DNA-protein structures that protect chromosome ends from degradation and shorten progressively with each cell division [1].

Telomere length measured in peripheral leukocytes has been used to determine an individual's "biological age", and previous reports have demonstrated that patients with chronic obstructive pulmonary disease (COPD) and/or emphysema have shorter peripheral leukocyte telomere length [2-5]. The presence of COPD and emphysema are independent risk factors for the ultimate development of lung cancer [6, 7].

The relationship between telomere length and lung cancer is controversial. Some reports have previously shown an increase in the risk for lung cancer development in subjects with shorter telomere length [8] but recent reports have described that smokers with longer peripheral leukocyte telomere length are also at increased risk [9].

The present study explored the potential independent association of peripheral leukocyte telomere length, COPD and emphysema with lung cancer diagnosis in a cross sectional analysis of a prospectively recruited population of active and former smokers participating in a lung cancer screening programme.

We included men and women of 40 years of age or older, who were current or former smokers with a smoking history of $\geqslant 10$ pack-years, and had no lung cancer symptoms from a lung cancer screening programme [6]. All patients underwent spirometry to determine the presence of airway obstruction (post bronchodilator value of forced expiratory volume in $1 \mathrm{~s}(\mathrm{FEV} 1) /$ forced vital capacity $(\mathrm{FVC})<0.70)$ and a low-dose chest computed tomography to determine the presence of emphysema (visually detected as yes versus no).

From the initial population of 1681 subjects participating (those with more than one visit) in our screening programme, we had blood samples from 1256 active or former smokers. Of them, 314 met the spirometric criteria for COPD. From this pool, we randomly selected 125 of them to participate in the present study. We matched each one of these patients with an active or former smoker without COPD of similar age ( \pm 2 years), sex, pack-years history ( \pm 3 pack-years), smoking status and body mass index (BMI) $\left( \pm 2 \mathrm{~kg} \cdot \mathrm{m}^{-2}\right)$. Relative telomere length was assessed in peripheral leukocytes using the comparative $\mathrm{Ct}$ method $(\mathrm{T} / \mathrm{S}=2-\Delta \Delta \mathrm{Ct})$ as previously described [10]. Cox regression analysis was used to explore the independent association of each predictive variable with lung cancer diagnosis. Predictor variables that were statistically significant $(\mathrm{p}<0.05)$ in the univariate analysis were included in a multiple Cox regression model to determine which of them independently related to lung cancer diagnosis. Calculations were made with SPSS version 20.0 Inc.

Patients included in the present study were mostly male (85\%), middle age (mean age: 60 years-old), slightly overweight (mean BMI between $27 \mathrm{Kg} \cdot \mathrm{m}^{-2}$ ), with a high pack-years history (mean 40 pack-years), and with a high percentage of them actively smoking (60 and 65\%). As expected from a matched population, no differences were found in age, sex, pack-years or smoking status history between groups. COPD patients had lower BMI and spirometric values than controls. 90\% of the COPD patients had mild-to-moderate airway obstruction. No differences were found in peripheral leukocyte telomere length between active and former smokers with and without COPD or in those with and without emphysema. During a median follow-up time of 52 months, a significantly higher proportion of patients with COPD were diagnosed with lung cancer ( $26 \%$ versus $5 \%$; $=0.001)$.

The univariate Cox analysis evaluating the independent association of each factor with a diagnosis of lung cancer is shown in table 1 . As expected, more pack-years, a lower BMI, the presence of COPD or of radiologically detected emphysema, were associated with lung cancer. Patients in the quartile characterised

@ERSpublications

Leukocyte telomere length should be included among the independent risk factors associated with lung cancer http://ow.ly/uzlg304Qpn6

Cite this article as: de-Torres JP, Sanchez-Salcedo P, Bastarrika G, et al. Telomere length, COPD and emphysema as risk factors for lung cancer. Eur Respir J 2017; 49: 1601521 [https://doi.org/10.1183/ 13993003.01521-2016]. 
by having the longest telomere length were most likely to have lung cancer. In the Cox multivariate analysis detailed in table 1, pack-years, the presence of COPD or of emphysema and longer telomere length quartile remained as independent predictors of lung cancer.

The present cross-sectional study showed that lung cancer screening participants with longer peripheral leukocyte telomere length have a higher risk of developing lung cancer, independent of the presence of COPD or radiological emphysema. This finding is interesting since these two diseases have been traditionally described as part of early and accelerated ageing processes associated with shorter telomere length [2] and increased lung cancer risk [6, 7].

JANG et al. [8] first postulated the link between shorter peripheral leukocytes telomere length and lung cancer risk. They studied 243 patients with lung cancer and 243 healthy controls of similar age, sex and smoking status and found that shorter telomere length (measured as quartiles or medians) was associated with a higher risk for lung cancer. MA et al. [11] later performed a meta-analysis, exploring a dataset of 11255 cases and 13101 controls from 21 publications showing also that shorter telomeres were significantly associated with a higher risk for lung cancer (OR 2.39, 95\% CI 1.18-4.88).

However, two recent studies have reported opposite findings. MACHIELA et al. [9] studied a group of 1536 individuals from the prospective Shanghai Women's Health Study (SWHS) and found that longer telomere length was associated with increased lung cancer risk (OR 1.51, 95\% CI 1.34-1.69). SEOW et al. [12] explored a pool analysis of subjects from the Prostate, Lung, Colorectal and Ovarian (PLCO) Cancer Screening Trial, the Alpha-Tocopherol, Beta-Carotene Cancer Prevention (ATBC) Trial and the SWHS and demonstrated that patients with the longest peripheral leukocyte telomere length had the highest risk for lung cancer. Importantly, none of these studies assessed the potential risk associated with the presence of COPD and/or emphysema. The current study provides a novel perspective of the most important risk factors associated with lung cancer by including into the analysis, the concurrent presence of COPD or emphysema as independent factors that could help explain the differences observed in previous studies.

Scarce information is available to explain the association between long telomere length and cancer risk. Recently, several reports have documented that mutations that lengthen telomeres are associated with increased risk of cancers, such as melanomas and gliomas. These reports suggest that the mechanism could relate to mutations in telomerase and sheltering genes, a mechanism called the "long telomere syndrome" [13]. The pathways are not yet fully defined but it seems to be responsible for certain histological types of cancers, with most information available regarding the development of melanomas.

TABLE 1 Univariate and multivariate Cox analysis exploring the independent association of the studied variables with lung cancer diagnosis in the entire population

\begin{tabular}{lccc} 
& p-value & HR & $\mathbf{9 5 \%} \mathbf{C l}$ \\
\hline Univariate analysis & & & \\
Age: for each year & 0.74 & 1.00 & $0.96-1.04$ \\
Sex: male versus female & 0.65 & 0.84 & $0.41-1.75$ \\
Pack-years: for each pack-year & 0.001 & 1.02 & $1.01-1.03$ \\
BMI: for each kg·m ${ }^{-2}$ & 0.03 & 0.92 & $0.85-0.99$ \\
Emphysema: yes versus no & 0.001 & 4.11 & $2.26-7.47$ \\
COPD: yes versus no & 0.001 & 4.23 & $2.34-7.63$ \\
Telomere length quartiles & & & \\
Q1: reference (shortest telomere length) & 0.001 & & \\
Q2 versus Q1 & 0.85 & 0.90 & $0.29-2.79$ \\
Q3 versus Q1 & 0.21 & 1.86 & $1.79-4.98$ \\
Q4 versus Q1 & 0.001 & 4.38 & $1.00-1.02$ \\
Multivariate analysis & & & $1.42-5.05$ \\
Pack-years: for each pack-year & 0.015 & 1.01 & $1.60-5.49$ \\
Emphysema: yes versus no & 0.002 & 2.68 & \\
COPD: yes versus no & 0.001 & 2.96 & \\
Telomere length quartiles & & & \\
Q1: reference (shortest telomere length) & 0.001 & $0.29-2.86$ \\
Q2 versus Q1 & 0.88 & 0.92 & $1.74-5.36$ \\
Q3 versus Q1 & 0.003 & 3.97 & \\
Q4 versus Q1 & & & $1.62-9.72$ \\
\hline
\end{tabular}

HR: hazard ratio; BMI: body mass index; COPD: Chronic obstructive pulmonary disease; Q: quartile. 
Interestingly, a recent report by ZHANG et al. [14] using tissue data on 51725 cases and 62035 controls, applied a Mendelian randomisation approach and estimated the associations between nine telomere length-associated SNPs and risk for five common cancer types (breast, lung, colorectal, ovarian and prostate cancer, including subtypes). They found that under Mendelian randomisation assumptions, longer telomere length increases the risk of lung adenocarcinoma. This information is important since $55 \%$ of our lung cancer cases are adenocarcinomas, in line with the hypothesis presented by these authors.

The present study had several limitations. Firstly, it is a cross sectional analysis and therefore cannot prove a cause-effect relationship. Secondly, the study was not designed to explore the mechanistic pathways behind this association. Future studies should explore telomerase activity measurement and sheltering gene mutations in trying to explain this novel finding. Thirdly, it is a surprise that we could not find differences in telomere length between smokers with and without COPD. The fact that this is a well-matched population of relatively young smokers with the same age, sex, pack-year history and smoking status, as well as the relatively small sample size, could explain the lack of differences in telomere length between the groups. However, this result further strengthens the results that show a significant association with the explored variables not due to emphysema or COPD.

In summary, active or former smokers participating in a lung cancer screening programme with peripheral leukocytes' telomere length in the longest quartile have an increased risk of lung cancer, independently of the presence of COPD or emphysema, two well-known lung cancer risk factors associated with shorter telomere length. Peripheral leukocyte telomere length should be included among the independent factor associated with lung cancer risk.

Juan P. de-Torres ${ }^{1}$, Pablo Sanchez-Salcedo ${ }^{1}$, Gorka Bastarrika ${ }^{2}$, Ana B. Alcaide ${ }^{1}$, Rubén Pío ${ }^{3}$, María Jose Pajares ${ }^{3}$, Arantza Campo ${ }^{1}$, Juan Berto ${ }^{1}$, Luis Montuenga ${ }^{3}$, Maria del Mar Ocon ${ }^{1}$, Carmen Monente ${ }^{1}$, Bartolome R. Celli ${ }^{4}$ and Javier J. Zulueta ${ }^{1}$

${ }^{1}$ Pulmonary Dept, Clínica Universidad de Navarra, Pamplona, Spain. ${ }^{2}$ Radiology Dept, Clínica Universidad de Navarra, Pamplona, Spain. ${ }^{3}$ Program in Solid Tumors and Biomarkers, Center for Applied Medical Research (CIMA), Pamplona, Spain. ${ }^{4}$ Pulmonary Dept, Brigham and Women's Hospital, Harvard Medical School Boston, Boston, MA, USA.

Correspondence: Juan P. de-Torres, Pulmonary Dept, Clínica Universidad de Navarra, Pamplona, Spain 31200. E-mail: jupa65@hotmail.com

Received: July 282016 | Accepted after revision: Sept 282016

Support statement: This work was supported in part by a grant (RD12/0036/0062) from Red Tematica de Investigacion Cooperativa en Cancer, Instituto de Salud Carlos III, Spanish Ministry of Economy and Competitiveness and European Regional Development Fund (ERDF) "Una manera de hacer Europa"; and by grants PI04/2404, PI07/0792, PI10/01652, PI11/01626, PI15/02157 Instituto de Salud Carlos III, Ministry of Economy and Competitiveness, Government of Spain. Funding information for this article has been deposited with the Open Funder Registry.

Conflict of interest: Disclosures can be found alongside this article at erj.ersjournals.com

Acknowledgements: J.P. de-Torres, G. Bastarrika, J.J. Zulueta, P. Sanchez-Salcedo, B.R. Celli (conception and design, analysis and interpretation, and drafting the manuscript for important intellectual content); A.B. Alcaide, J. Berto, A. Campo, M. del Mar Ocon, C. Monente, R. Pío, M.J. Pajares, L. Montuenga (analysis and interpretation).

\section{References}

1 Blackburn EH, Greider CW, Szostak JW. Telomeres and telomerase: the path from maize, Tetrahymena and yeast to human cancer and aging. Nat Med 2006; 12: 1133-1138.

2 Mercado N, Ito K, Barnes PJ. Accelerated ageing of the lung in COPD: new concepts. Thorax 2015; 70: 482-489.

3 Albrecht E, Sillanpää E, Karrasch S, et al. Telomere length in circulating leukocytes is associated with lung function and disease. Eur Respir J 2014; 43: 983-992.

4 Savale L, Chaouat A, Bastuji-Garin S, et al. Shortened telomeres in circulating leukocytes of patients with chronic obstructive pulmonary disease. Am J Respir Crit Care Med 2009; 179: 566-571.

5 Rode L, Bojesen SE, Weischer M, et al. Short telomere length, lung function and chronic obstructive pulmonary disease in 46,396 individuals. Thorax 2013; 68: 429-435.

6 Sanchez-Salcedo P, Berto J, de-Torres JP, et al. Lung cancer screening: fourteen year experience of the Pamplona early detection program (P-IELCAP). Arch Bronconeumol 2015; 51: 169-176.

7 Wilson DO, Weissfeld JL, Balkan A, et al. Association of radiographic emphysema and airflow obstruction with lung cancer. Am J Respir Crit Care Med 2008; 178: 738-744.

8 Jang JS, Choi YY, Lee WK, et al. Telomere length and the risk of lung cancer. Cancer Sci 2008; 99: 1385-1389.

9 Machiela MJ, Hsiung CA, Shu XO, et al. Genetic variants associated with longer telomere length are associated with increased lung cancer risk among never-smoking women in Asia: a report from the female lung cancer consortium in Asia. Int J Cancer 2015; 137: 311-319.

10 Cawthon RM. Telomere measurement by quantitative PCR. Nucleic Acids Res 2002; 30: e47.

$11 \mathrm{Ma} \mathrm{H}$, Zhou Z, Wei S, et al. Shortened telomere length is associated with increased risk of cancer: a meta-analysis. PLoS One 2011; 6: e20466.

12 Seow WJ, Cawthon RM, Purdue MP, et al. Telomere length in white blood cell DNA and lung cancer: a pooled analysis of three prospective cohorts. Cancer Res 2014; 74: 4090-4098. 
13 Stanley SE, Armanios M. The short and long telomere syndromes: paired paradigms for molecular medicine. Curr Opin Genet Dev 2015; 33: 1-9.

14 Zhang C, Doherty JA, Burgess S, et al. Genetic determinants of telomere length and risk of common cancers: a Mendelian randomization study. Hum Mol Genet 2015; 24: 5356-5366.

Copyright @ERS 2017 